\title{
Dissociating Memory Retrieval Processes using fMRl: Evidence that Priming Does Not Support Recognition Memory
}

\author{
D.I. Donaldson, ${ }^{1,7}$ S.E. Petersen, ${ }^{2,3,4,5}$ \\ and R.L. Buckner $2,4,5,6$ \\ ${ }^{1}$ Department of Psychology \\ University of Stirling \\ Stirling \\ United Kingdom \\ ${ }^{2}$ Department of Psychology \\ ${ }^{3}$ Department of Neurology \\ ${ }^{4}$ Department of Radiology \\ ${ }^{5}$ Department of Anatomy and Neurobiology \\ ${ }^{6}$ Howard Hughes Medical Institute \\ Washington University \\ St. Louis, Missouri 63108
}

\section{Summary}

We employed event-related fMRI to constrain cognitive accounts of memory retrieval. Studies of explicit retrieval reveal that lateral and medial parietal, dorsal middle frontal gyrus, and anterior prefrontal cortex respond more for studied than new words, reflecting a correlate of "retrieval success." Studies of implicit memory suggest left temporal cortex, ventral and dorsal inferior frontal gyrus respond less for studied than new words, reflecting a correlate of "conceptual priming." In the present study, responses for old and new items were compared during performance on explicit recognition (old/new judgement) and semantic (abstract/concrete judgement) tasks. Regions associated with priming were only modulated during the semantic task, whereas regions associated with retrieval success were modulated during both tasks. These findings constrain functional-anatomic accounts of the networks, suggesting that processes associated with priming do not support explicit recognition judgments.

\section{Introduction}

Human memory is thought to be supported by multiple retrieval processes. Dual process theories posit that memory tasks such as old/new recognition associated with explicit or conscious remembering rely upon two retrieval mechanisms: recollection, which is an effortful search-like process, and familiarity, which is a more automatic process (cf. Atkinson and Juola, 1973; Gardiner and Java, 1993; Jacoby and Dallas, 1981; Mandler, 1980). A separate phenomenon, priming, is facilitated processing due to prior exposure to a stimulus, such as improved performance or reduced reaction times, and can occur even in the absence of explicit remembering (cf. Roediger and McDermott, 1993; Tulving and Schacter, 1990; Schacter, 1994). Dissociating these processes, and understanding the relations between them, has proved difficult with traditional behavioral measures.

Here fMRI was employed to examine the relation between explicit familiarity, and implicit priming, in particu-

${ }^{7}$ Correspondence: did1@stir.ac.uk lar conceptual priming (cf. Jacoby and Dallas, 1981; Mayes, 1991). Depending on task requirements, both perceptual and conceptual processing can be primed (associated with the sensory form and the meaning of the stimulus, respectively) (cf. Blaxton, 1989; Roediger, 1990). Behavioral (cf. Wagner, Gabrieli, and Verfaellie, 1997) and neuropsychological (cf. Stark and Squire, 2000) evidence suggests that perceptual priming does not contribute toward familiarity during performance on explicit retrieval tasks. However, whether memory processes associated with conceptual priming support explicit retrieval remains unclear. Described below are findings from imaging data that suggest this question can be addressed using functional anatomical measures.

Event-related fMRI studies of explicit recognition reveal a network of brain regions whose activity provides an index of episodic "retrieval success" (cf. Henson et al., 1999; Konishi et al., 2000; McDermott et al., 2000; Donaldson et al., 2001; see also Habib and Lepage, 1999; Nyberg et al., 1995; Rugg et al., 1996; Schacter et al., 1996; Nolde et al., 1998; Wagner et al., 1998). Left and right lateral inferior parietal cortex, medial parietal cortex (precuneus), left dorsal middle frontal gyrus, and left anterior prefrontal cortex exhibit a larger transient hemodynamic response for old than new items. Moreover, activity within these regions is reduced when subjects fail to recognize old items relative to correctly recognized items (cf. Sanders et al., 2000). In short, the magnitude of response in these regions is predictive of successful retrieval.

Studies using tasks that involve repeated semantic judgments on words reveal a separate network of regions, associated with "conceptual priming." Ventral and dorsal left inferior frontal gyrus and left temporal cortex exhibit reduced activity for old compared to new items - the opposite pattern to that associated with episodic retrieval success (cf. Demb et al., 1995; Buckner et al., 1998a, 2000; Gabrieli et al., 1996; Schacter and Buckner, 1998; and see Buckner and Koutstaal, 1998 for findings from amnesic subjects). Direct comparisons of conceptual (e.g., abstract/concrete judgments) and perceptual (e.g., uppercase/lowercase judgments) tasks reveal that left inferior frontal cortex only exhibits priming effects during performance of conceptual tasks (cf. Demb et al., 1995; see also Blaxton et al., 1996). Moreover, priming reductions seen in these regions are amodal, occurring for both visual and auditory stimuli (cf. Buckner et al., 2000), a finding that is particularly hard to reconcile with a perceptual priming account. In short, these effects are associated with facilitation in the maintenance/manipulation and use/evaluation of semantic and/or lexical information, and provide a neural correlate of conceptual priming.

The present study investigates the relationship between these neuroanatomically distinct networks, using fMRI in a hypothesis-driven way. The finding that the two networks described above are known to be modulated by different memory retrieval processes is central to this approach. However, it is not assumed that these 


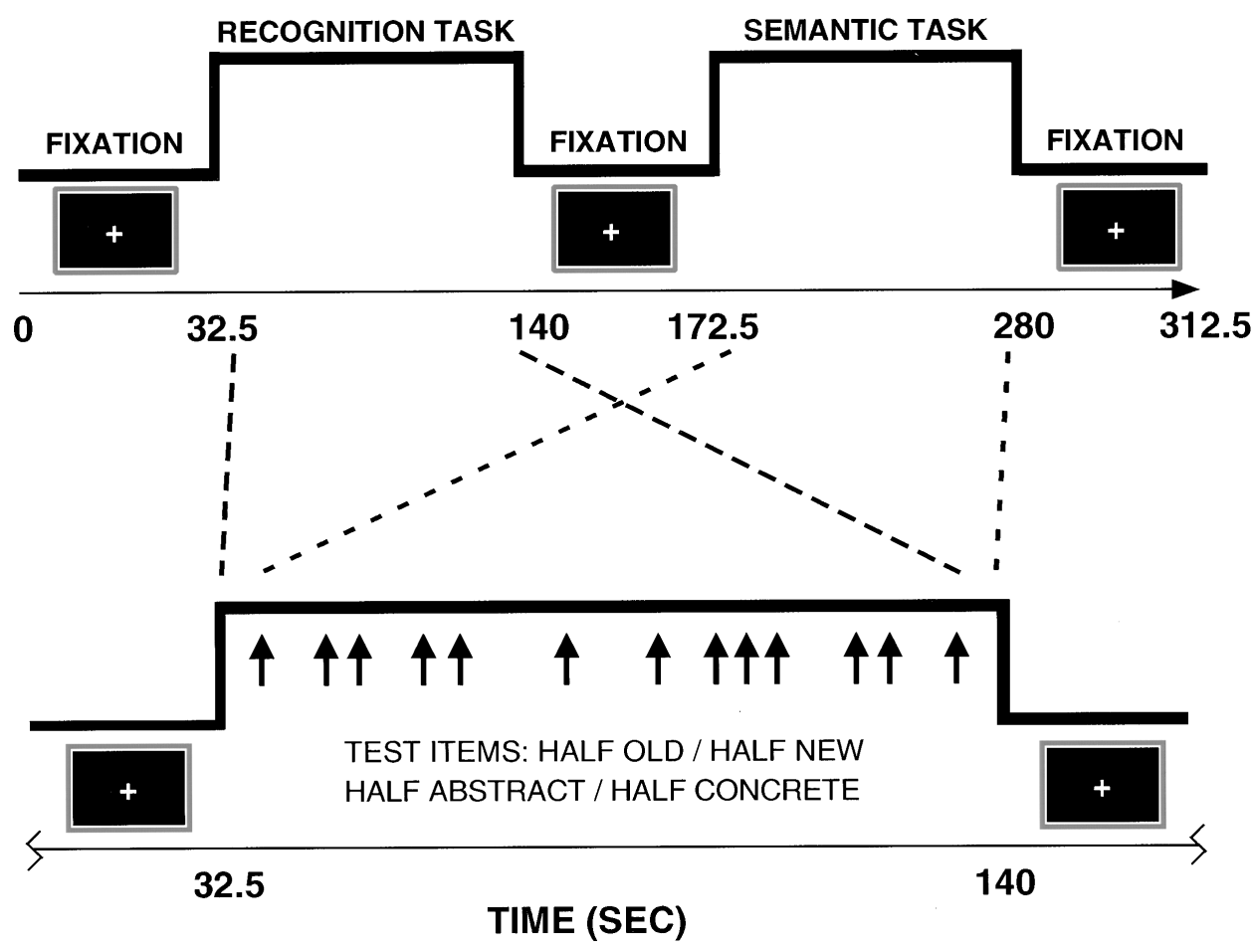

Figure 1. The Mixed Blocked and Event-Related Experimental Design Used during a Single fMRI Run

Subjects performed multiple study-test sessions, with scanning only occurring at test. This figure shows the structure of a single test session. Top: at test, each functional run was blocked; subjects alternated between fixating on a cross-hair and performing the recognition and semantic judgment tasks. Task order was alternated across scans. Bottom: within each memory block, subjects were presented with temporally jittered test items.

are the only regions modulated by these particular retrieval processes, or that these regions support memory retrieval processes exclusively. Rather, the approach rests on the demonstration that activity within each set of regions provides an index of a particular retrieval process. Two specific questions are pursued. (1) Can the two networks described above can be functionally dissociated? And from a cognitive perspective, (2) to what degree do these separate networks contribute to performance on explicit memory tasks such as old/new recognition-a judgment that relies in part on familiarity based recognition processes.

At study, subjects made semantic judgments to words using an abstract/concrete judgment task (Demb et al., 1995). At test, old and new words were presented and subjects alternated between the same semantic (abstract/concrete) task and an old/new recognition task. As Figure 1 shows, each task was presented in a discrete block, separated by blocks of a baseline task (fixation), but within each task block, the test items were jittered and pseudo-randomized using event-related procedures. This mixed "blocked and event-related" design allows the transient item-related responses to old and new test words to be examined while controlling for possible sustained differences in subjects' overall cognitive set or state (cf. Donaldson et al., 2001; Donaldson and Buckner, 2001).

Using measures of activity within the networks described above, we ask whether regions that exhibit conceptual priming reductions during the semantic task show similar reductions during the old/new recognition task. Activity increases associated with explicit retrieval success were also explored to understand their relation to conceptual priming effects and to ask whether they can support recognition in the absence of any priming associated activity reductions. If regions associated with conceptual priming show no reduction during the recognition task, this would suggest that conceptual priming does not support the contribution of familiarity to recognition memory.

\section{Results}

\section{Behavioral}

Recognition memory performance showed a mean hit rate of $91 \%$ and false alarm rate of $13 \%$. Reaction time data revealed that responses were significantly faster for hits than correct rejections (across subject means of $863 \mathrm{~ms}$ and $954 \mathrm{~ms}$, respectively; $t[21]=4.56, p<$ 0.001). Examining responses to all old and new test items during the recognition task, mean reaction times were faster for old than new items (988 ms and 1035 $\mathrm{ms}$, respectively). However, this difference did not reach significance $(p>0.1)$. By contrast, a clear priming effect was observed in the semantic task; significantly reduced reaction times for old compared to new words (869 ms and $946 \mathrm{~ms}$, respectively; $t[21]=6.83, p<0.0001$ ).

\section{Imaging}

Statistical whole-brain activation maps were formed to examine the old minus new difference for each task. Items were not divided based on performance accuracy 

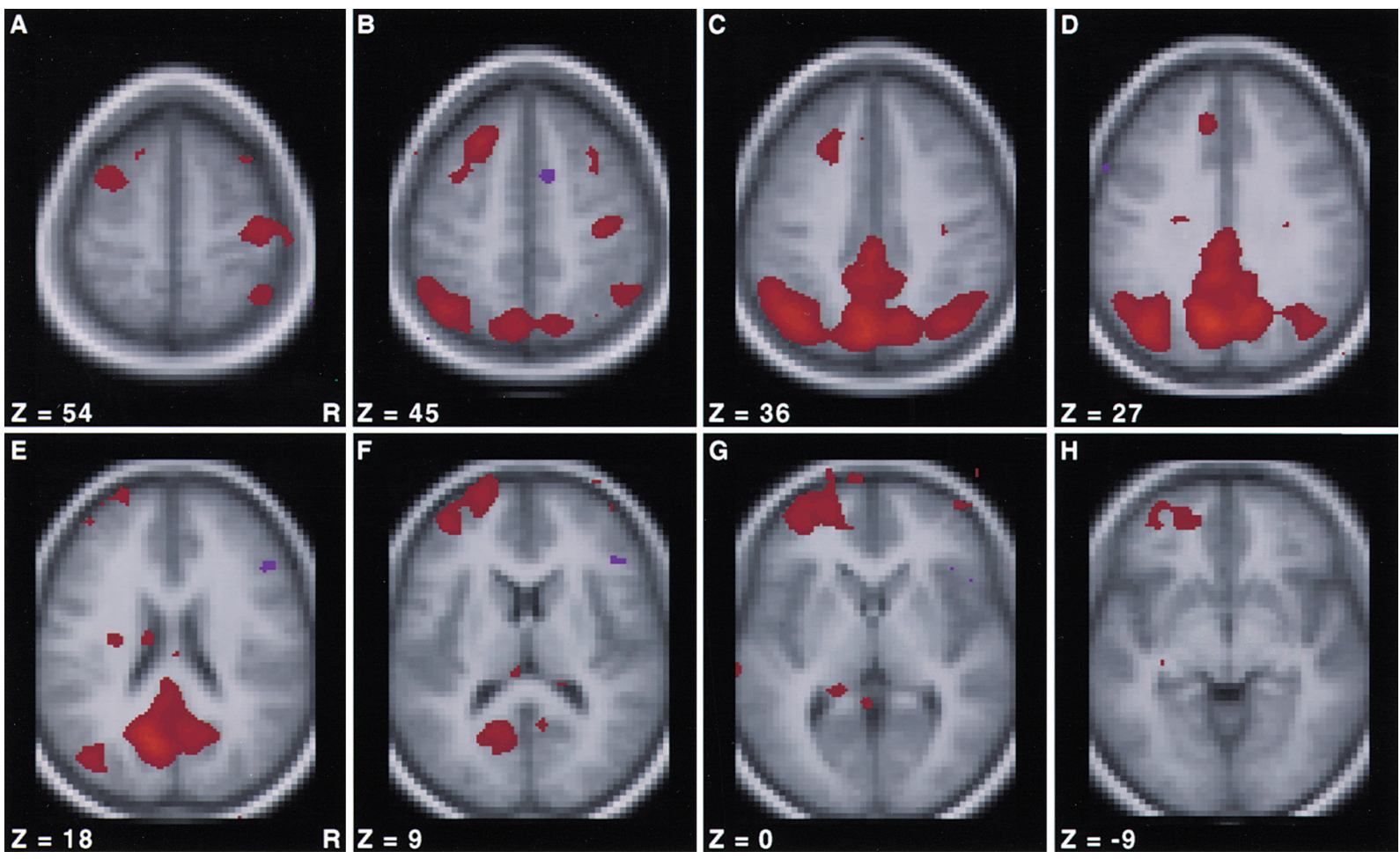

Figure 2. Recognition Task

Statistical activation maps showing regions of significantly different transient activity for old than new test items, superimposed onto average structural brain images. Regions showing increased activity for old relative to new items are shown in red, regions showing decreased activity in blue. Significant activation peaks are listed in Table 1.

(e.g., hits versus correct rejections) because this would not allow equivalent comparison between the episodic and semantic tasks. However, because recognition performance was high, old items were almost always hits and new items were almost always correct rejections. Recognition Task Activation Maps

Figure 2 shows regions that exhibit a significant difference between old and new test items during the explicit recognition task. A network of areas previously shown to be related to retrieval success during recognition memory was observed, including left and right lateral parietal cortex, medial parietal cortex (precuneus), left dorsal middle frontal gyrus, and left anterior prefrontal cortex (at or near Brodmann area 10). The regions of activation match closely those seen in previous eventrelated studies of retrieval success (cf. Donaldson et al., 2001; Konishi et al., 2000), replicating these findings. The location of activated regions, identified by peaks, are listed in Table 1 (both positive and negative differences are listed).

\section{Semantic Task Activation Maps}

Figure 3 shows regions that exhibit a significant difference between old and new test items during performance of the semantic judgment task. Two sets of regions are clear. The same network of areas that was more active for old than new stimuli during the recognition task is also seen for the semantic task, including left and right lateral parietal cortex, medial parietal cortex (precuneus), left dorsal middle frontal gyrus, and anterior prefrontal cortex. In addition, a second set of regions, similar to that previously observed to correlate with con- ceptual priming (cf. Buckner et al., 2000; Wagner et al., 2000; Schacter and Buckner, 1998), shows the opposite effect, with less activation for old than new test stimuli, including left ventral (at or near BA 44/6) and dorsal (at or near BA 44/45/47) inferior frontal gyrus, and left temporal cortex. The location of activated regions, identified by peaks, are listed in Table 2 (both positive and negative differences are listed).

\section{Time Course Data}

The time course of activation was examined in a hypothesis-driven fashion based on previous findings (see Introduction). Targeted analyses were conducted using a random effects statistical analysis, testing the specific hypotheses outlined in the Introduction and exploring the temporal dynamics of the old/new effects. Two sets of a priori regions were defined as being retrieval success or conceptual priming regions, based on the locations of peak activation points taken from studies in which these memory effects have been described previously (taken from Donaldson et al., 2001; and Buckner et al., 2000, respectively). This approach has the potential to reduce the size of any effects that are found because a priori regions are unlikely to exactly match the maximum activation points in the present data. It does, however, strengthen confidence in any dissociation found between the two tasks.

All time course data are displayed as subtraction waveforms-showing differences in hemodynamic response between old and new test items. This allows the size and direction of the effects of prior exposure to be seen clearly, highlighting features that distinguish 
Table 1. Activation Peaks for Old-New Difference during the Recognition Task

\begin{tabular}{|c|c|c|c|c|}
\hline \multicolumn{3}{|c|}{ Coordinates } & \multicolumn{2}{|c|}{ Significance } \\
\hline$x$ & $Y$ & $z$ & Z Score & BA/Area \\
\hline \multicolumn{5}{|c|}{ Old $>$ New } \\
\hline-7 & -69 & 30 & 8.66 & 19 \\
\hline-43 & -63 & 42 & 6.96 & $19 / 40$ \\
\hline-37 & -69 & 33 & 6.95 & $19 / 39$ \\
\hline 10 & -66 & 30 & 6.63 & 19 \\
\hline-7 & -45 & 30 & 6.30 & $7 / 31$ \\
\hline-4 & -33 & 33 & 6.24 & 31 \\
\hline 40 & -21 & 63 & 5.89 & $3 / 4$ \\
\hline 37 & -69 & 36 & 5.63 & $19 / 39$ \\
\hline-25 & 24 & 45 & 5.42 & 8 \\
\hline 37 & 48 & 9 & 5.00 & 46 \\
\hline-22 & 60 & 9 & 4.77 & 10 \\
\hline 34 & -21 & 48 & 4.65 & $3 / 4$ \\
\hline-10 & 36 & 30 & 4.5 & 9 \\
\hline 40 & -51 & 54 & 4.12 & $7 / 40$ \\
\hline 34 & -69 & -36 & 3.99 & Cerebellum \\
\hline-31 & 9 & 54 & 3.96 & 6 \\
\hline-13 & -12 & 18 & 3.92 & Thalamus \\
\hline-19 & -39 & 3 & 3.90 & Hippocampus \\
\hline 25 & -33 & -24 & 3.86 & Cerebellum \\
\hline-13 & 48 & -12 & 3.83 & Cerebellum \\
\hline-7 & -48 & -21 & 3.70 & Cerebellum \\
\hline 40 & 57 & 6 & 3.65 & 10 \\
\hline \multicolumn{5}{|c|}{ Old $<$ New } \\
\hline 7 & 6 & 48 & 4.15 & 6 \\
\hline 46 & 27 & 18 & 3.81 & 45 \\
\hline
\end{tabular}

Coordinates are listed in Talairach and Tournoux (1988) atlas space. BA is the Brodmann area nearest to the coordinates and should be considered approximate.

priming from retrieval success effects. Figure 4 shows how a positive hemodynamic response that is larger for old than new stimuli results in a positive going subtraction waveform (top). If no difference in response exists between old and new items, then the subtraction waveform is flat, suggesting no modulation based on previous experience (middle). Finally, if the positive going hemodynamic response is reduced for old compared to new words, then the subtraction results in a negative going waveform (bottom). Although other combinations of responses could give rise to similar waveforms (e.g., combinations of negative going hemodynamic responses), each of the time courses described below follows one of the patterns illustrated in Figure 4.

Five regions (left dorsal middle frontal gyrus $(-37,6$, $54)$, left anterior prefrontal cortex $(-40,51,6)$, left lateral inferior parietal lobe $(-40,-51,39)$, medial parietal cortex $(-1,-63,39)$, and right lateral inferior parietal lobe $(49,-45,48))$ that have exhibited retrieval success effects during episodic memory were constructed based on peak coordinates taken from Donaldson et al. (2001). In that study, only correct responses were analyzed, hence the term retrieval success. Namely, each region is shown in Figure 5, alongside a time course waveform for that region, showing the difference in activity between old and new stimuli for both recognition (black) and semantic (red) tasks. In each case, the time course reveals a hemodynamic response profile that exhibits a positive increase in activity for old compared to new stimuli. The response typically begins around $2.5 \mathrm{~s}$ poststimulus, and peaks around 5 or $7.5 \mathrm{~s}$ before returning to baseline. Although these regions are identified as retrieval success regions associated with episodic memory retrieval, Figure 5 indicates that they are also sensitive to the difference between old and new stimuli during performance of the semantic judgment task. In left dorsal middle frontal gyrus, left anterior prefrontal cortex, and left lateral inferior parietal lobe, the response appears to be approximately equivalent for both tasks. By contrast, the old minus new differences appear to be bigger for semantic than recognition judgment in medial parietal cortex and right lateral inferior parietal lobe.

Three regions were identified that exhibit conceptual priming effects during tasks that involve semantic/lexical judgments, taken from Buckner et al. (2000). Namely, left dorsal $(-43,9,34)$ and ventral $(-43,34,3)$ inferior frontal gyrus, and left temporal cortex $(-43,-46,6)$. Each of the regions previously associated with conceptual priming is identified in Figure 6, shown alongside the time course of activity in that region. As in Figure 5, the time course data show the difference between old and new stimuli for both recognition (black) and semantic (red) tasks. As would be expected on the basis of previous studies, these regions show a reduction in activity for old compared to new stimuli during the semantic judgment task. In each region, the effect begins around $2.5 \mathrm{~s}$ poststimulus, and peaks around $5 \mathrm{~s}$ before returning to baseline. By contrast, these regions reveal little or no difference in response to old and new stimuli during performance of the recognition task; the waveforms are essentially flat, with no clear hemodynamic response. Thus, regions previously associated with retrieval success effects are modulated by prior exposure to an item during both recognition and semantic judgment tasks, whereas regions previously associated with conceptual priming effects are only sensitive to prior exposure during the semantic judgment task, where both the task and items repeat from study to test.

\section{Dissociation between Conceptual Priming}

\section{and Retrieval Success Networks}

To provide further statistical support for the apparent dissociation between the two networks, we employed random effects ANOVA. This analysis compared the difference in response to old and new stimuli, in each set of regions, across the two tasks. Activity was averaged across the regions in each network, with the regions defined on an a priori basis as being associated with either priming or retrieval success. A single estimate was extracted for the combined average of the retrieval success regions and the conceptual priming regions, and these measures were employed in an ANOVA, testing for an interaction between behavioral task and neural network. ANOVA revealed a significant network (conceptual priming versus retrieval success) by task (episodic versus semantic) interaction $(F[1,22]=5.98, p<$ 0.05 ). As can be seen in Figure 7, this result is consistent with the account of the time course data described above. Subsidiary analyses confirmed that the retrieval success network exhibited a significant positive increase for old compared to new items in both the recognition and semantic judgment tasks $(t[21]=3.04, p<$ 0.005 , and $t[21]=3.71, p<0.001$, respectively). More- 

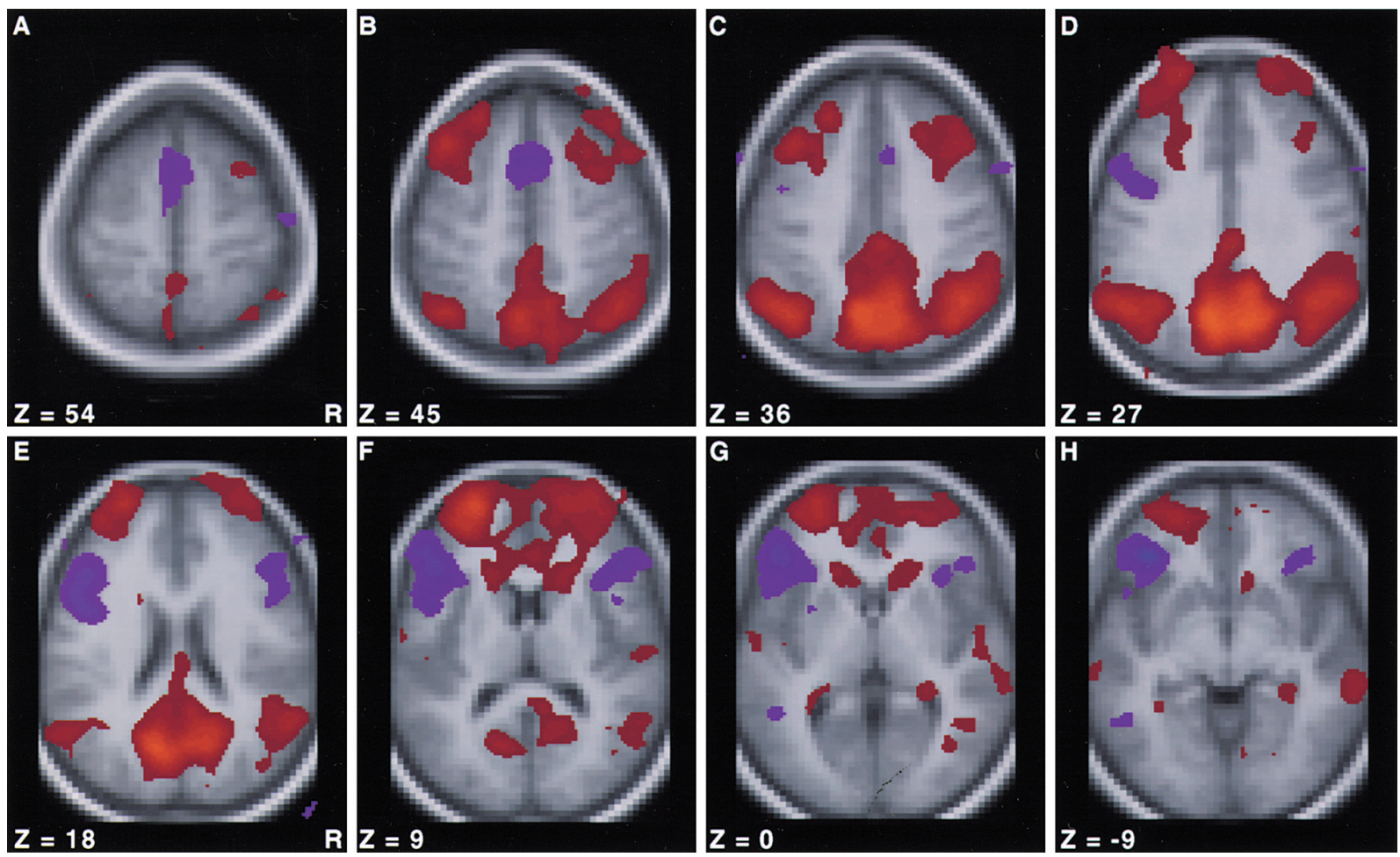

Figure 3. Semantic Task

Statistical activation maps showing regions of significantly different transient activity for old than new test items, superimposed onto average structural brain images. Regions showing increased activity for old relative to new items are shown in red, regions showing decreased activity in blue. Significant activation peaks are listed in Table 2.

over, the effect was of similar magnitude for both task conditions $(0.06 \%$ and $0.07 \%$ for the recognition and semantic tasks, respectively) with no significant difference between them ( $t[21]=0.36, p=0.73)$. By contrast, for the priming network, the old items showed a significant reduction relative to the new items that was significant for the semantic task $(t[21]=2.59, p<0.01)$, but not for the recognition task $(t[21]=0.22, p=0.42)$, with a significant difference between the two tasks $(t[21]=$ $2.21, p<0.05)$. In sum, these regional analyses confirm the dissociation between the networks associated with conceptual priming and retrieval success. As Figure 7 makes clear, the old/new effect associated with conceptual priming was near to zero during the recognition task $(-0.0026 \%$, compared to $-0.05 \%$ during the semantic task), suggesting that it played minimal, if any, role in supporting performance in the recognition task.

Analysis Based on Reaction Time

One basis for distinguishing between recollection and familiarity is response time, with familiarity generally thought to occur more rapidly than recollection (cf. Hintzman and Curran, 1994). Certain forms of recollection, associated with the retrieval of noncriterial information or associative information, may also occur relatively quickly (cf. Yonelinas and Jacoby, 1996). Nonetheless, comparison of the magnitude of the retrieval success effects in fast and slow recognition trials provides some degree of constraint on the kind of retrieval processes supported. Specifically, if retrieval success effects are only present for slow responses, this would suggest that they are more likely associated with controlled recollection processes than familiarity. For this analysis, a within-subject median-split across trials was conducted for each of the old and new trials in the recognition task. The magnitude of activation in the combined retrieval success regions was then computed for fast and slow trials and entered into ANOVA.

ANOVA did not reveal a significant difference in the retrieval success regions between fast and slow decisions $(t[21]=1.16, p=0.26$, effects with a magnitude of $0.06 \%$ and $0.08 \%$, respectively). Nonetheless, analysis revealed that the effect was independently significant for both the fast and slow recognition responses $(t[21]=$ $3.83, p<0.001$, and $t[21]=2.63, p<0.01$, respectively). The presence of comparable effects for both fast and slow responses does not provide strong evidence for a specific association with either recollection or familiarity. Of course, this finding could reflect little more than the fact that slow responses are associated with increases in both recollection and familiarity relative to fast responses. Regardless, the analyses suggest that the effect is not specifically associated with recollection processes that are engaged when decisions rely solely on slow, extended, controlled, or search-like processing.

\section{Discussion}

Neural correlates of processes supporting explicit and implicit memory retrieval were identified and dissociated using fMRI. First, a network of regions (lateral and medial 
Table 2. Activation Peaks for Old-New Difference during the Semantic Judgment Task

\begin{tabular}{|c|c|c|c|c|}
\hline \multicolumn{3}{|c|}{ Coordinates } & \multicolumn{2}{|c|}{ Significance } \\
\hline$x$ & $Y$ & z & Z Score & BA/Area \\
\hline \multicolumn{5}{|c|}{ Old $>$ New } \\
\hline-10 & -66 & 30 & 12.48 & $18 / 31$ \\
\hline 10 & -66 & 30 & 11.62 & $18 / 31$ \\
\hline-25 & 57 & 12 & 9.65 & 10 \\
\hline 40 & -57 & 36 & 8.80 & $19 / 39$ \\
\hline-46 & -63 & 36 & 8.49 & $19 / 39$ \\
\hline-40 & 24 & 45 & 7.45 & 8 \\
\hline-25 & 60 & 24 & 6.52 & 10 \\
\hline-25 & 51 & -3 & 6.24 & 10 \\
\hline 16 & 60 & 12 & 5.92 & 10 \\
\hline 1 & -27 & 33 & 5.77 & $31 / 23$ \\
\hline-25 & 36 & 45 & 5.66 & 8 \\
\hline-37 & 51 & 24 & 5.43 & $9 / 10$ \\
\hline 37 & 24 & 36 & 5.31 & 9 \\
\hline 37 & 33 & 45 & 4.90 & 8 \\
\hline 31 & 9 & 48 & 4.77 & $6 / 8$ \\
\hline-7 & 51 & 6 & 4.75 & $32 / 10$ \\
\hline 7 & -45 & 15 & 4.66 & 30 \\
\hline 31 & 42 & 9 & 4.54 & 46 \\
\hline-28 & 15 & 36 & 4.36 & 9 \\
\hline 22 & 27 & 42 & 4.28 & 8 \\
\hline 25 & -39 & 3 & 4.27 & Hippocampus \\
\hline 4 & 54 & -15 & 4.22 & 11 \\
\hline-31 & -48 & -3 & 4.18 & Lingual Gyrus \\
\hline 52 & -18 & 6 & 4.12 & 21 \\
\hline-55 & -12 & -39 & 3.92 & Cerebellum \\
\hline 37 & -57 & 9 & 3.89 & 19 \\
\hline 10 & 18 & -3 & 3.82 & Caudate \\
\hline 37 & -69 & 3 & 3.76 & $19 / 37$ \\
\hline 52 & -12 & -27 & 3.51 & 20 \\
\hline-61 & -9 & 9 & 3.50 & 42 \\
\hline \multicolumn{5}{|c|}{ Old $<$ New } \\
\hline-37 & 30 & -9 & 8.87 & 47 \\
\hline-46 & 24 & 18 & 7.35 & $44 / 45$ \\
\hline-46 & 6 & 21 & 7.13 & 44 \\
\hline-46 & 33 & 3 & 7.00 & $44 / 45 / 47$ \\
\hline-4 & 15 & 54 & 6.43 & 6 \\
\hline 43 & 27 & 15 & 6.30 & $44 / 45$ \\
\hline 34 & 30 & -6 & 4.53 & $44 / 45 / 47$ \\
\hline 34 & 21 & 9 & 4.41 & $44 / 45 / 47$ \\
\hline 46 & 12 & 21 & 4.30 & $44 / 45$ \\
\hline 58 & 42 & 18 & 3.92 & $45 / 46$ \\
\hline-46 & -51 & 0 & 3.88 & 37 \\
\hline-34 & -48 & -21 & 3.60 & Cerebellum \\
\hline
\end{tabular}

Coordinates are listed in Talairach and Tournoux (1988) atlas space. $B A$ is the Brodmann area nearest to the coordinates and should be considered approximate.

parietal cortex, left dorsal middle frontal gyrus, and anterior prefrontal cortex, cf. Figure 2) exhibited more transient activity in response to old than new test items during both the recognition and semantic judgment tasks. Second, a separate network of regions (left dorsal and ventral inferior frontal gyrus and left temporal cortex, cf. Figure 3) exhibited less transient activity in response to old than new test items during the semantic judgment task but not the recognition task.

In addition to the two networks being neuroanatomically distinct, the difference between old and new items occurs in the opposite directions between networks, and the networks are functionally dissociable, with modulation in the conceptual priming regions being depen- dent upon task demands. The implications of these findings are discussed below, interpreted in the context of current models of memory retrieval. We suggest that these data demonstrate that regions sensitive to implicit memory processes associated with priming were not modulated during, and thus did not support, explicit memory processes associated with retrieval success in the recognition task.

Explicit Memory: Retrieval Success Effects

The findings from the recognition memory task replicate event-related fMRI findings reported previously (see Figures 2 and 5; cf. Henson et al., 1999; Konishi et al., 2000; McDermott et al., 2000; Donaldson et al., 2001). A network of regions in parietal and frontal cortex is sensitive to whether an item is old or new during the recognition task. The presence of significant modulations within this network during the semantic judgment task was unexpected; subjects were not instructed to remember the words. Support for this finding comes from a recent study by Koutstaal et al. (2000), who reported similar effects during performance on a semantic task involving judgments of the size of objects.

Based on the current functional account of these regions as indexing retrieval success, the present findings suggest that subjects may have experienced some level of explicit memory during the semantic judgment task. Indeed, given the experimental conditions employed in the present study, it seems likely that subjects consciously recognized at least some of the old items presented during the semantic retrieval blocks, i.e., that there were explicit intrusions (cf. Bowers and Schacter, 1990; Schacter, 1994). Thus, we are inclined to add a simple caveat to the retrieval success account. Namely, for the memory processes reflected in these regions to be engaged, subjects need not be required to actively seek to retrieve episodic information in the pursuit of current task demands (see also Koutstaal et al., 2000).

By this account, the retrieval success effect supports recognition memory performance, but the mechanism can be obligatorily or unintentionally engaged rather than always being controlled or driven by current task demands - at least under circumstances where welllearned stimuli are paired with strong retrieval cues. In relation to cognitive accounts of recognition memory, a description of the effect as obligatory suggests an explicit retrieval mechanism that fits the functional characteristics of an automatic familiarity process more than a controlled strategy-based recollective process. Evidence lending weight to this possibility comes from the analysis of the retrieval success effect based on reaction time. The retrieval success effect was similar in magnitude for fast and slow recognition decisions, a finding that is difficult to reconcile with an account solely in terms of a slow search-like recollection process. Moreover, the fact that retrieval success regions are independent of regions showing state-related activity associated with top-down "retrieval mode" processes provides additional support to this conclusion (cf. Donaldson et al., 2001). Finally, we stress that there is good evidence that these effects reflect a retrieval mechanism that supports recognition performance; the size of the response in these regions is predictive of successful retrieval during recognition memory (cf. Sanders et al., 2000). 
ORIGINAL SUBTRACTION

WAVEFORMS WAVEFORMS

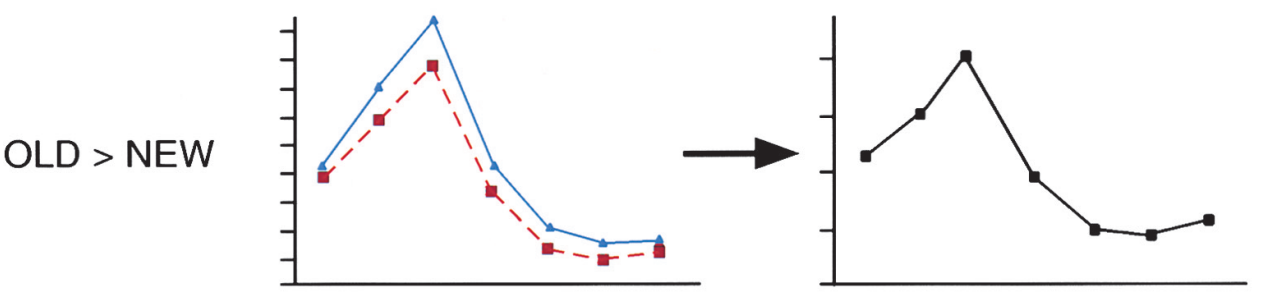

OLD $=$ NEW

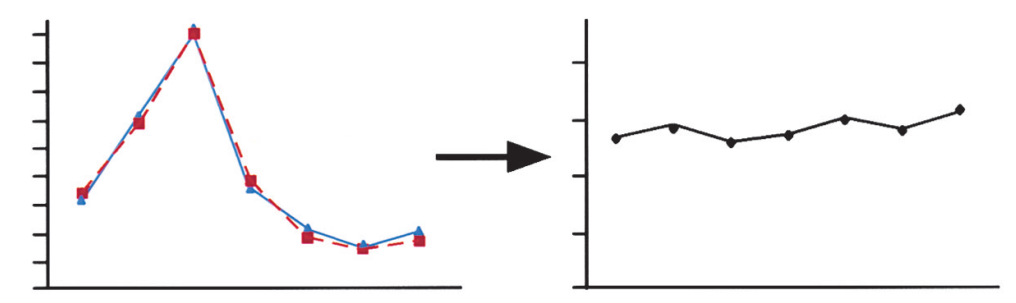

OLD $<$ NEW

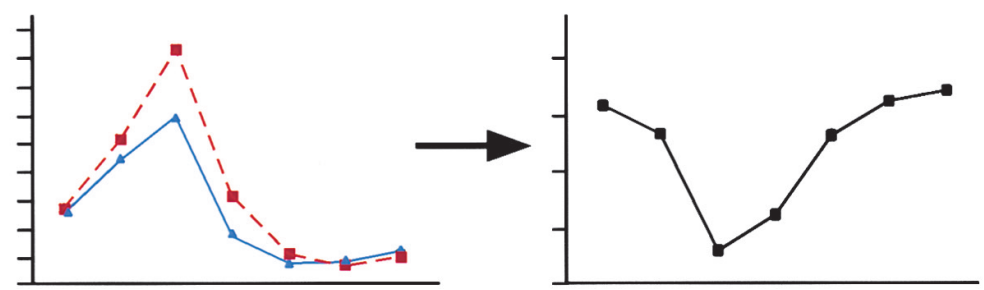

Figure 4. Generating Subtraction Waveforms

The figure illustrates how the hemodynamic responses to old and new test items are used to generate subtraction waveforms (red = old, blue $=$ new, black = old minus new). Top: a larger positive response to old than new items gives rise to a positive going subtraction waveform. Middle: equivalent response to old and new items gives rise to a flat subtraction waveform, suggesting no modulation based on previous experience with test items. Bottom: a smaller positive response to old than new items gives rise to a negative going subtraction waveform.

Implicit Memory: Conceptual Priming Effects

A second network, including left dorsal and ventral inferior frontal gyrus and left temporal cortex, was found to exhibit behavior typically associated with priming (i.e., reduced activity for old compared to new words, see Figures 3 and 6; cf. Demb et al., 1995; Buckner and Koutstaal, 1998; Buckner et al., 1998a, 2000). The present data add weight to the conceptual priming account of these regions, but highlight an important constraint, process specificity. Priming-related modulations were only present when both the item and the task were repeated - minimal priming reductions were found in the recognition task in this network of regions. This finding suggests that the benefit of previously experiencing an item is selective, operating in a process-specific manner. By this account, priming will occur if a task requires the recapitulation of processing, as is suggested by a "transfer appropriate processing" view (cf. Morris et al., 1977; Blaxton, 1989; Roediger et al., 1989).

Support for a transfer appropriate view of the conceptual priming effects can be found in Wagner et al. (2000; see also Schacter and Buckner, 1998). The manipulation used in the present study, and that of Wagner et al., can be characterized in terms of a between- versus within-task study-test manipulation. However, we stress that it is not task repetition per se that is important. Rather, priming effects should be seen to the degree that study and test tasks call upon equivalent processing of a repeated item. Consistent with this account, recent behavioral evidence suggests that different forms of conceptual priming are revealed depending on the task employed (cf. Vaidya et al., 1997; Gabrieli et al., 1999). This possibility also raises a suggestion for why activity reductions were noted across tasks in the present study: the study and test procedures were the same in the semantic task and differed in the old/new recognition task.

On the Relationship between Processes Supporting Explicit and Implicit Memory Retrieval

As noted above, regions that exhibit priming-related reductions during the semantic task were not modulated by previous experience during performance of the rec- 


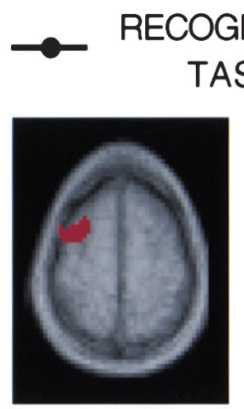

$(-37654)$

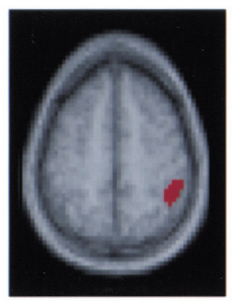

$(49-4548)$

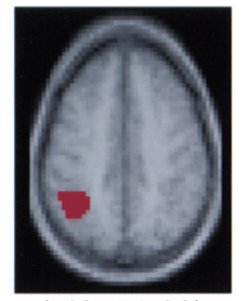

$(-40-5139)$

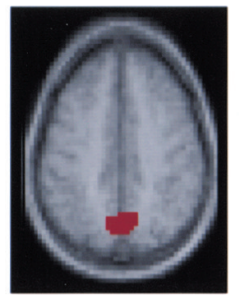

$(-1-6339)$

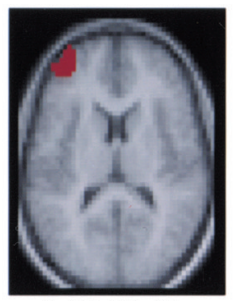

$(-40516)$
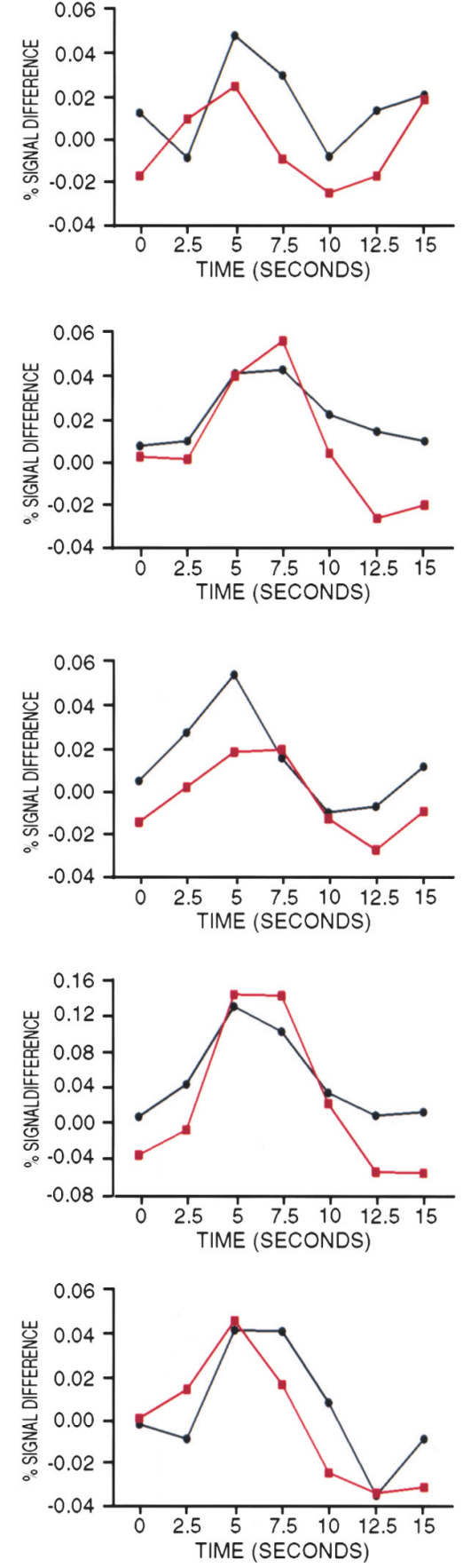

Figure 5. Retrieval Success Network

Left: statistical activation maps showing regions of interest. Regions shown are (from top to bottom) left dorsal middle frontal gyrus, right lateral parietal, left lateral parietal, medial parietal, and anterior prefrontal cortex. Right: time course of the difference in transient hemodynamic response to old and new test items for regions identified in the activation maps. Activity is expressed as the difference in percent signal change between old and new items. The old minus new difference is shown for both recognition (black) and semantic judgment (red) tasks. For each region, robust responses are evident for both tasks. ognition task (see Figures 2 and 6). This finding is important in two respects. First, it adds weight to the general hypothesis that processes supporting implicit and explicit memory are functionally and neurally dissociable. Second, it speaks to current debate about the possible relation between priming and familiarity outlined in the Introduction. In short, we believe that the present data support the hypothesis that conceptual priming does not contribute to familiarity processes underlying successful recognition memory performance.

Behavioral (cf. Wagner et al., 1997) and neuropsychological (cf. Hamann and Squire, 1997; Stark and Squire, 2000) studies provide evidence that perceptual priming mechanisms do not contribute toward the familiarity process associated with episodic recognition judgments. By contrast, the behavioral and neuropsychological evidence does not speak to the role of conceptual priming in recognition memory. For example, Wagner et al. (1997, p320) ask whether the same process "mediates explicit recognition and conceptual priming." The data presented here address this issue. Regions sensitive to priming were not significantly modulated during, and thus could not support, recognition memory performance. In terms of cognitive models of recognition memory, this finding provides strong evidence that conceptual priming does not support the contribution of familiarity to successful memory retrieval during recognition memory. As noted above, however, different forms of conceptual priming may exist (cf. Vaidya et al., 1997; Gabrieli et al., 1999) and further investigation is required to determine whether these are associated with activation in the same or different brain regions.

Two important a priori assumptions underlie this conclusion. First, the network of regions exhibiting old/new effects during the semantic task condition does indeed reflect the operation of a conceptual priming mechanism. Theoretically, regions exhibiting increased activity for old than new items during the semantic task could reflect processing associated with priming. There is considerable evidence in support of the assumption that priming is associated with decreases in activity (see Introduction; cf. Schacter and Buckner, 1998, for a review). Second, our interpretation rests on the assumption that performance on the recognition memory task was associated, to some degree, with familiarity. It could be argued that performance was based entirely or largely upon recollection, in which case priming effects associated with familiarity would be absent or minimal. As noted above, the retrieval success regions were modulated by both fast and slow recognition responses, a finding that is difficult to reconcile with a controlled recollection account of the retrieval success effects. Moreover, recent accounts of recognition memory suggest that performance typically involves a combination of recollection and familiarity (cf. McElree, Dolan, and Jacoby, 1999), and it seems likely that this is the case here.

Nonetheless, it should be noted that experimental tasks can almost certainly be constructed such that conceptual priming effects are correlated with successful episodic memory retrieval. For example, this would be the case if a two-stage judgment had been required with both semantic and episodic task components or if study-test overlap was more pronounced in a recognition test. In the present study, the processing requirements 


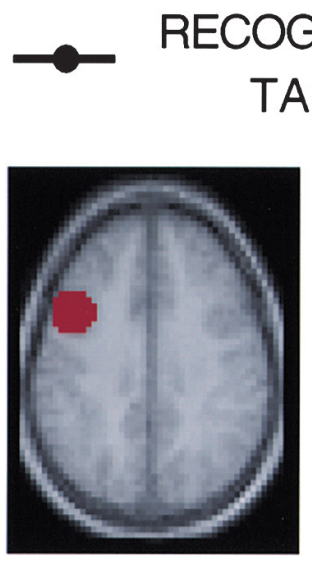

$(-43934)$

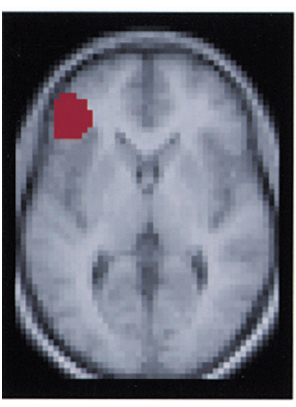

$(-43343)$

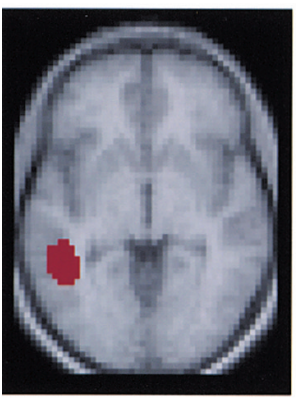

$(-43-46-6)$
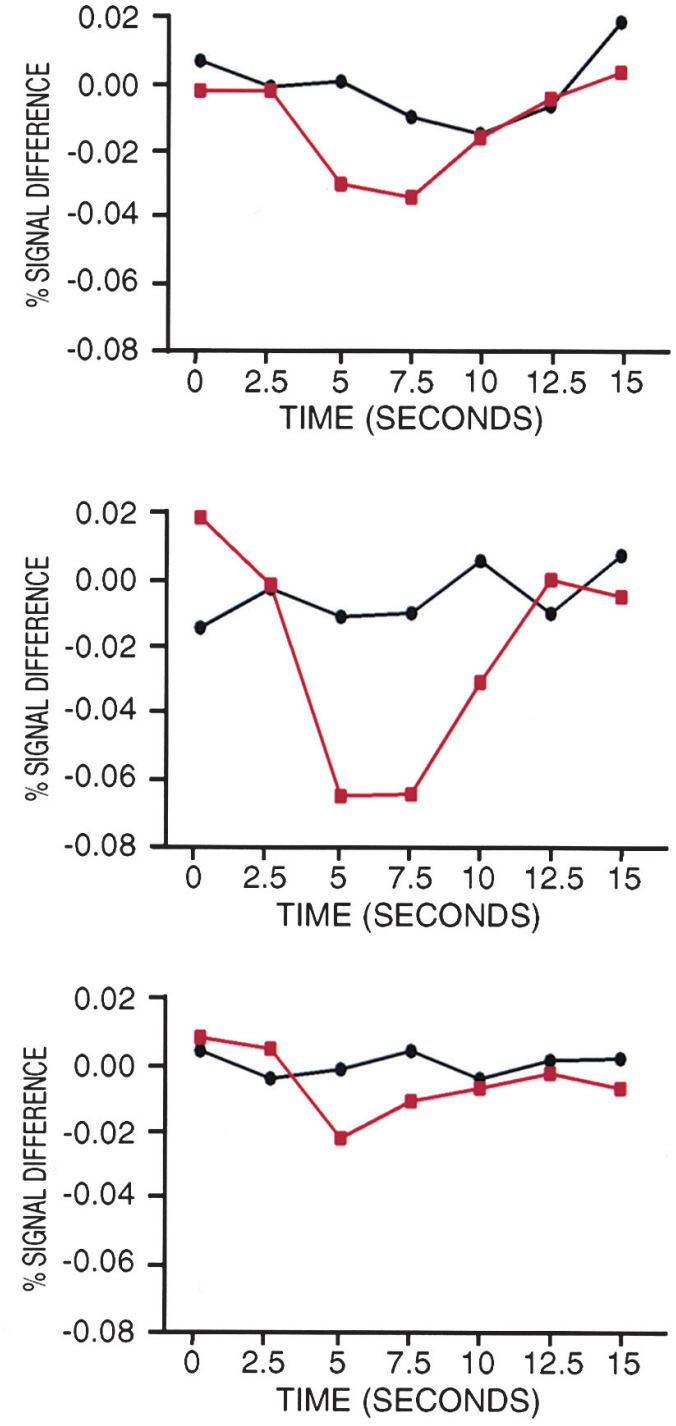

Figure 6. Conceptual Priming Network Left: statistical activation maps showing regions of interest. Regions shown are (from top to bottom) left ventral inferior frontal gyrus, left dorsal inferior frontal gyrus, and left inferior temporal cortex. Right: time course of the difference in transient hemodynamic response to old and new test items for regions identified in the activation maps. Activity is expressed as the difference in percent signal change between old and new items. The old minus new difference is shown for both recognition (black) and semantic (red) tasks. For each region, a robust response is evident for the semantic task only, with little or no modulation for the recognition task. were only matched at study and test for the semantic task. Repeating the semantic judgment presumably led to priming effects because of the overlap in processing demands, whereas switching to a recognition task at test did not. Thus, the present findings support the conclusion that under typical episodic memory testing conditions, conceptual priming effects associated with implicit memory do not support explicit recognition memory. However, it seems plausible that, when no information is available from the explicit retrieval success network to support task performance and study-test overlap is sufficient, it may be possible for subjects to resort to the use of other sources of information in making recognition judgements, including conceptual priming effects.

Finally, we highlight two important caveats. First, the absence of a modulation within priming-sensitive regions during performance of the recognition task could reflect little more than "component overlap," that is, a reduction due to priming that occurs at the same time as, and in the same regions as, an enhancement due to other processing demands. Although logically possible, this seems unparsimonious and somewhat unlikely - opposing effects would have to match near perfectly to cancel out. Second, the absence of an effect could reflect a type I error. Although null effects should be considered with caution, the findings presented here represent the most bounded, and therefore most interpretable, form of null result. The subtraction time courses presented in Figure 6 are essentially flat for the recognition task, representing a mean difference of zero. By contrast, priming effects were present and detectable during performance on the semantic task, under conditions of similar power. Moreover, regional analyses showed the effect to be near zero when data were pooled across regions, an approach that 

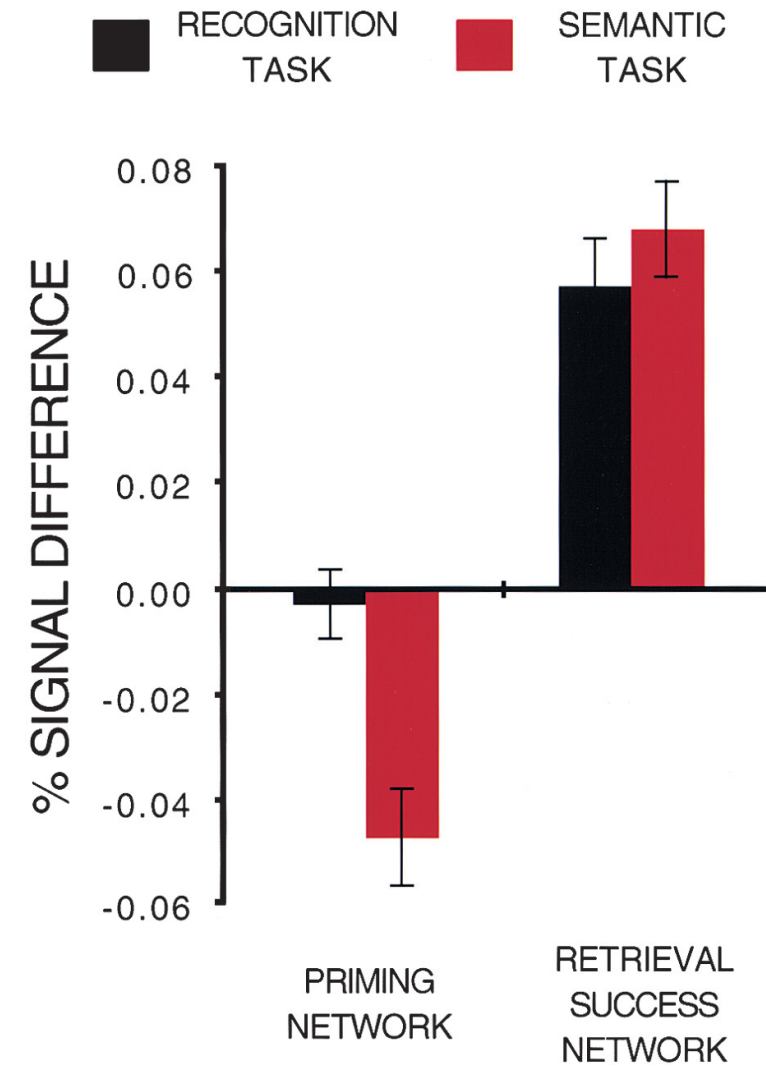

Figure 7. Dissociating Implicit and Explicit Memory

The figure shows the magnitude (mean percent signal change and standard error bars) of the difference between old and new test items within the conceptual priming and retrieval success networks. The dissociation is 2-fold. First, equivalent effects are present for the recognition (black) and semantic judgment (red) tasks within the retrieval success network, whereas significant effects are only found in the conceptual priming network during the semantic task. Second, the difference between old and new items is positive going in the retrieval success network but negative going in the conceptual priming network. This interaction provides strong support for a functional-anatomic dissociation between the memory processes associated with these two networks of regions.

provides considerable power (see Figure 7). In short, the results likely reflect the true absence of an effect, rather than the failure to find an effect that is present.

\section{Summary}

fMRI was employed to test and constrain cognitive accounts of memory retrieval. The dissociation presented here between two cortical networks is 2-fold; differences in the functional characteristics of the regions (i.e., which tasks gave rise to old versus new modulations) and differences in the pattern of behavior exhibited by each network (i.e., more or less activity in response to old and new test stimuli). The present findings highlight the importance of characterizing the underlying temporal profile of neural activity, beyond simply assessing whether a difference exists. Perhaps the most important aspect of the findings, however, is that they address the relation between retrieval processes, providing functional-anatomic evidence that conceptual priming does not contribute toward processes support- ing successful explicit memory retrieval. This finding adds weight to current behavioral and neuropsychological evidence for a distinction between implicit memory processes associated with priming and the explicit memory process of familiarity that supports performance on recognition memory tasks.

\section{Experimental Procedures}

\section{Subjects and Materials}

Twenty-four subjects (seven male; mean age 22, range 18-32 years; right-handed, native English speakers, with normal vision, and no reported neurological problems) from the Washington University community participated for a $\$ 75$ payment. Informed consent was obtained in accordance with the guidelines and approval of the Washington University Human Studies Committee. Data from two subjects were excluded due to excessive movement artifacts. Behavioral stimuli consisted of 336 nouns and verbs, 4-8 letter long; half abstract/half concrete based on ratings from Paivio, Yuille, and Madigan (1968). Mapping of stimuli to item type (old versus new) and task (recognition versus semantic judgment) was counterbalanced across subjects. Stimuli were presented in central vision, in Geneva font, in white capital letters on a black background, and subtended approximately 0.5 degrees of visual angle per letter.

\section{Data Acquisition}

MRI data were acquired using a Siemens 1.5 Tesla Vision System (Erlangen, Germany). T1-weighted structural images were acquired first (MP-RAGE sequence: TR $=9.7 \mathrm{~ms}, \mathrm{TE}=4 \mathrm{~ms}$, flip angle = $10^{\circ}, \mathrm{TI}=20 \mathrm{~ms}, \mathrm{TD}=500 \mathrm{~ms}$, voxel size $=1 \times 1 \times 1.25 \mathrm{~mm}$ ). Functional images were acquired using an asymmetric spin-echo echo-planar sequence sensitive to blood oxygenation level-dependent (BOLD) contrast $\left(\mathrm{T} 2^{\star}\right)\left(\mathrm{TR}=2.5 \mathrm{~s}, \mathrm{~T}^{*}\right.$ evolution time $=50$ $\mathrm{ms}$, voxel size $=3.75 \times 3.75 \mathrm{~mm}$ in-plane resolution). Pillows and thermoplastic facemasks minimized head movement, headphones dampened scanner noise and enabled communication. A power Macintosh computer (Apple, Cupertino, CA) and Psyscope software (Cohen et al., 1993) controlled stimulus display and recorded responses from a fiber-optic key-press device. An LCD projector (AmPro model LCD-150) projected stimuli onto a screen at the head of the scanner, viewable via a mirror attached to the coil. Subjects performed six functional runs during which 128 sets of 16 contiguous $8 \mathrm{~mm}$-thick axial images were acquired parallel to the anteriorposterior commissure plane. The first four images in each run allowed stabilization of longitudinal magnetization; these images were used to facilitate alignment, but were excluded from analysis of the functional data.

\section{Behavioral Paradigm}

Each functional run was preceded by an unscanned study session using an incidental encoding task. A list of 28 words (half abstract, half concrete) was presented twice. Using a $2.5 \mathrm{~s}$ intertrial interval, each word was displayed for $750 \mathrm{~ms}$, followed by a fixation crosshair $(+)$ for the remainder of the trial. Subjects judged quickly and accurately whether each word was abstract or concrete, responding with a button press. A scanned test session was then performed, during which subjects alternated between three tasks, fixating on a cross-hair, old/new recognition, and semantic (abstract/concrete) judgments. As Figure 1 shows, the tasks were blocked. At the onset of each block, a visual prompt ("fixation," "old-new," or "abstractconcrete") was displayed for $2 \mathrm{~s}$, indicating which task to perform. During fixation blocks, a cross-hair was displayed continuously for subjects to fixate on.

Each scanned functional run lasted approximately 320 s (128 acquisitions, 1 acquisition every $2.5 \mathrm{~s}, 3$ acquisitions occurring prior to the onset of the first task block), separated by a 3 min break during which the next study session was performed. The order of recognition and semantic judgment tasks was counterbalanced across runs. Fixation blocks lasted $30 \mathrm{~s}$ (equivalent to the presentation of 12 trials at $2.5 \mathrm{~s}$ per trial), task blocks lasted $105 \mathrm{~s}$ (equivalent to the presentation of 42 trials at $2.5 \mathrm{~s}$ per trial). During the episodic task block, 14 old (half abstract, half concrete) word trials, 14 new 
(half abstract, half concrete) word trials, and 14 fixation trials were presented. Similarly, during the semantic task block, an identical mix of stimuli were presented; 14 old (half abstract, half concrete) word trials, 14 new (half abstract, half concrete) word trials, and 14 fixation trials. The presentation of test items was time-locked to the onset of successive whole-brain image acquisitions. Each test item was displayed for $750 \mathrm{~ms}$, followed by a cross-hair for the remainder of the $2.5 \mathrm{~s}$ trial. Test items presented during memory task blocks were jittered by interspersing gaps (i.e., the fixation trials) throughout the blocks, such that short fixation periods occurred during the task blocks as well as during the continuous fixation blocks. Trial order within each block was pseudo-randomized so that each type of event (presentation of old, new, and fixation trials) was equally likely to follow each other, using procedures described in Buckner et al. (1998a).

Recognition and semantic judgment task blocks were identical other than in the instructions given to subjects. During recognition task blocks, subjects were required to discriminate between old (studied) and new (unstudied) words. During semantic task blocks, subjects judged whether each word was abstract or concrete (the same task performed at study). Subjects were told that the test items would be a mixture of abstract and concrete words during the recognition task, but that their task was just to judge whether or not the words were old. Similarly, they were told that the test items would be a mixture of old and new words during the semantic task, but that their task was simply to judge whether the words were abstract or concrete. For both tasks, responses were to be made as quickly and accurately as possible, using the first fingers of the left and right hands. The mapping of fingers to old and new and to abstract and concrete responses was counterbalanced across subjects.

\section{Data Analysis}

Data from each subject was preprocessed to remove noise and artifacts, including: (a) correction for movement within and across runs using a rigid-body rotation and translation algorithm (cf. Snyder, 1996), (b) whole brain normalization to a common mode of 1000 to allow for comparisons across subjects (cf. Ojemann et al., 1997), and (c) temporal realignment (using sinc interpolation) of all slices to the temporal midpoint of the first slice, accounting for differences in the acquisition time of each individual slice. Data were then resampled into $3 \mathrm{~mm}$ isotopic voxels, warped into standardized atlas space (cf. Talairach and Tournoux, 1998), and smoothed with a gaussian filter (6 mm FWHM).

Preprocessed data were analyzed using the General Linear Model (GLM; Friston et al., 1994; Josephs et al., 1997; Zarahn et al., 1997) on a voxel-by-voxel basis. Estimates of the magnitude of effects were derived from the model. Transient effects (i.e., responses to old and new test items) were accounted for by coding the seven time points (17.5 s) immediately following each stimulus onset. Sustained effects were also coded into the GLM to account for differences in the baseline between semantic and recognition tasks (cf. Donaldson et al., 2001; Donaldson and Buckner, 2001). For each task, this was done by coding the sustained effect as a boxcar function lasting the duration of the task block, convolved with a hemodynamic response function accounting for the rise and fall of the hemodynamic response (cf. Boynton et al., 1996). Coding the sustained task effects controls for any differences in mental set between the two tasks, ensuring that any differences in the transient response to the individual test items are not influenced by differences in the overall level of sustained activity during each task. The sustained effects are to be reported separately, do not influence any of the conclusions drawn here, and are therefore considered effects of no interest for the purpose of the present paper. Factors were also coded to account for the visual prompts at the beginning of each block, low frequency noise effects (a high pass filter with a cutoff frequency of $.009 \mathrm{~Hz}$ ), and the within-run linear trend.

Average z-statistical maps were derived from the GLM. For the transient effects, model estimates were cross-correlated with a series of five lagged hemodynamic response functions (each separated by 1 s; cf. Boynton, et al., 1996; Dale and Buckner, 1997), accounting for possible variation in the onset of the hemodynamic response (cf. Schacter et al., 1997; Buckner et al., 1998b). All effects are described in terms of percent signal change, defined as signal magnitude divided by the mean signal intensity across all functional runs after the components of linear drift and coded effects were removed. This mean is given by the average over all runs of the intercept term of the linear trend. Peak coordinate locations listed in Tables 1 and 2 were derived from the activation maps, and were generated using a statistical threshold of 6 or more voxels above $p<$ 0.001 (note that each voxel $=3 \mathrm{~mm}^{3}$ ). This threshold is equivalent to that used previously by Buckner et al. (1998a) and was verified to yield few false positives. When multiple peaks occurred within $12 \mathrm{~mm}$ of each other, the peak with the highest $\mathrm{z}$ value was kept.

Statistical analyses examined differences between old and new test items. Whole-brain activation maps were formed to examine the old minus new difference for each task. In addition, random effects statistical analysis was performed, using regions of interest defined a priori as being retrieval success or conceptual priming regions, based on the locations of peak activation points taken from studies in which memory effects have been found previously (taken from Donaldson et al., 2001, and Buckner et al., 2000, respectively). For each seed point, a region was defined within the combined statistical map of transient responses to both old and new stimuli during both episodic and semantic tasks. Specifically, all voxels within $12 \mathrm{~mm}$ of the peak location that were more significant than $p<0.001$ were included in the region. For each region, the difference in hemodynamic response between old and new test items was extracted for both the recognition and semantic tasks; the mean magnitude (in percent signal change, defined above) of the response to old and new test items was extracted at each of seven poststimulus time points. For statistical tests based on a random effect analysis, the estimated amplitude of the response was extracted for each region for each subject, based on the difference between peak (third) and baseline (first) time points.

\section{Acknowledgments}

We thank Laura Williams and Seiki Konishi for help with data collection, Endel Tulving, Patrick Dolan, David Gallo, Larry Jacoby, Margaret Sheridan, Mark Wheeler, Todd Braver, Deanna Barch, Jeff Zacks, and Henry Roediger for helpful discussion. This work was funded by NIH grants MH57506 (R.L.B.) and NS32979 (S.E.P.), the McDonnell Center for Higher Brain Function (R.L.B.), a James S. McDonnell Foundation Program in Cognitive Neuroscience Grant (R.L.B.), and a Wellcome Trust International Traveling Research Fellowship (D.I.D.).

Received February 20, 2001; revised June 25, 2001.

\section{References}

Atkinson, R.C., and Juola, J.F. (1973). Factors influencing speed and accuracy of word recognition. In Fourth International Symposium on Attention and Performance, S. Kornblum, ed. (New York: Academic Press), pp. 583-612.

Boynton, G.M., Engel, S.A., Glover, G.H., and Heeger, D.J. (1996). Linear systems analysis of functional magnetic resonance imaging in human V1. J. Neurosci. 16, 4207-4221.

Bowers, J.S., and Schacter, D.L. (1990). Implicit memory and test awareness. J. Exp. Psychol. Learn. Mem. Cogn. 16, 404-416.

Blaxton, T.A. (1989). Investigating dissociations among memory measures: support for a transfer-appropriate processing framework. J. Exp. Psychol. Learn. Mem. Cogn. 15, 657-668.

Blaxton, T.A., Bookheimer, S.Y., Zeffiro, T.A., Figlozzi, C.M., and Gaillard, W.D. (1996). Functional mapping of human memory using PET: comparisons of conceptual and perceptual tasks. Can. J. Exp. Psychol. 50, 42-56.

Buckner, R.L., and Koutstaal, W. (1998). Functional neuroimaging studies of encoding, priming, and explicit memory retrieval. Proc. Natl. Acad. Sci. USA 95, 891-898.

Buckner, R.L., Goodman, J., Burock, M., Rotte, M., Koutstaal, W., Schacter, D., Rosen, B.R., and Dale, A.M. (1998a). Functional-anatomic correlates of object priming in humans revealed by rapid presentation event-related fMRI. Neuron 20, 285-296.

Buckner, R.L., Koutstaal, W., Schacter, D.L., Dale, A.M., Rotte, M., and Rosen, B.R. (1998b). Functional-anatomic study of episodic 
retrieval. II. Selective averaging of event-related fMRI trials to test the retrieval success hypothesis. Neuroimage 7, 163-175.

Buckner, R.L., Koutstaal, W., Schacter, D.L., Wagner, A.D., and Rosen, B.R. (2000). Functional MRI evidence for a role of frontal and inferior temporal cortex in amodal components of priming. Brain 123, 620-640.

Cohen, J.D., MacWhinney, R.C., Flatt, M., and Provost, J. (1993). Psyscope: An interactive graphic system for designing and controlling experiments in the psychology laboratory using Macintosh computers. Behav. Res. Methods Instrument Comput. 25, 257-271.

Dale, A.M., and Buckner, R.L. (1997). Selective averaging of rapidly presented individual trials using fMRI. Hum. Brain Mapp. 5, 329-340. Demb, J.B., Desmond, J.E., Wagner, A.D., Vaidya, C.J., Glover, G.H., and Gabrieli, J.D.E. (1995). Semantic encoding and retrieval in the left inferior prefrontal cortex: A functional MRI study of task difficulty and process specificity. J. Neurosci. 15, 5870-5878.

Donaldson, D.I., Petersen, S.E., Ollinger, J.M., and Buckner, R.L. (2001). Dissociating state- and item-related processes of recognition memory using fMRI. Neuroimage 13, 129-142.

Donaldson, D.I., and Buckner, R.L. (2001). Effective paradigm design. In Functional Magnetic Resonance Imaging of the Brain: Methods for Neuroscience, P.M. Matthews, P. Jezzard, and A.C. Evans, eds. (Oxford: Oxford University Press), in press.

Friston, K.J., Jezzard, P., and Turner, R. (1994). The analysis of functional MRI time series. Hum. Brain Mapp. 1, 153-171.

Gabrieli, J.D.E., Desmond, J.E., Demb, J.B., Wagner, A.D., Stone, A.V., Vaidya, C.J., and Glover, G.H. (1996). Functional magnetic resonance imaging of semantic memory processes in the frontal lobes. Psychol. Sci. 7, 278-283.

Gabrieli, J.D.E., Vaidya, C.J., Stone, M., Francis, W.S., ThompsonSchill, S.L., Fleischman, D.A., Tinklenberg, J.R., Yesavage, J.A., and Wilson, R.S. (1999). Convergent behavioral and neuropsychological evidence for a distinction between identification and production forms of repetition priming. J. Exp. Psychol. Gen. 128, 479-498.

Gardiner, J.M., and Java, R.I. (1993). Recognising and remembering. In Theories of Memory, A. Collins, S.E. Gathercole, M.A. Conway, and P.E. Morris, eds. (Mahwah, NJ: Lawrence Erlbaum Associates), pp. 163-188.

Habib, R., and Lepage, M. (1999). Novelty assessment in the brain. In Memory, Consciousness and the Brain, E. Tulving, ed. (Philadelphia, PA: Psychology Press), pp. 265-277.

Hamann, S.B., and Squire, L.R. (1997). Intact priming for novel perceptual representations in amnesia. J. Cogn. Neurosci. 9, 699-713. Henson, R.N.A., Rugg, M.D., Shallice, T., Josephs, O., and Dolan, R.J. (1999). Recollection and familiarity in recognition memory: An event-related functional magnetic resonance imaging study. J. Neurosci. 15, 3962-3972.

Jacoby, L.L., and Dallas, M. (1981). On the relationship between autobiographical memory and perceptual learning. J. Exp. Psychol. Gen. 110, 306-340.

Josephs, O., Turner, R., and Friston, K. (1997). Event-related fMRI. Hum. Brain Mapp. 5, 243-248.

Konishi, S., Wheeler, M.E., Donaldson, D.I., and Buckner, R.L. (2000). Neural correlates of retrieval success. Neuroimage 12, 276-286.

Koutstaal, W., Wagner, A.D., Rotte, M., Maril, A., Buckner, R.L., and Schacter, D.L. (2000). Perceptual specificity in visual object priming: functional magnetic resonance imaging evidence for a laterality difference in fusiform cortex. Neuropsychologia 39, 184-199.

Hintzman, D.L., and Curran, T. (1994). Retrieval dynamic of recognition and frequency judgments: Evidence for separate processes of familiarity and recall. J. Mem. Lang. 33, 1-18.

Mandler, G. (1980). Recognising: The judgment of previous occurrence. Psychol. Rev. 87, 252-271.

Mayes, A.R. (1991). Automatic memory processes in amnesia: How are they mediated? In The Neuropsychology of Consciousness, A.D. Milner and M.D. Rugg, eds. (San Diego, CA: Academic Press Ltd). McDermott, K.B., Jones, T.C., Petersen, S.E., Lageman, S.K., and Roediger, H.L. (2000). Retrieval success is accompanied by enhanced activation in anterior prefrontal cortex during recognition memory: An event-related fMRI study. J. Cogn. Neurosci. 12, 965-976.

McElree, B., Dolan, P.O., and Jacoby, L.L. (1999). Isolating the contributions of familiarity and source information to item recognition: A time course analysis. J. Exp. Psychol. Learn. Mem. Cogn. 25, 563-582.

Morris, C.D., Bransford, J.D., and Franks, J.J. (1977). Levels of processing versus transfer appropriate processing. J. Verbal Learn. Verbal Behav. 16, 519-533.

Nolde, S.F., Johnson, M.K., and Raye, C.L. (1998). The role of prefrontal cortex during tests of episodic memory. Trends Cognit. Sci. 2, 399-406.

Nyberg, L., Tulving, E., Habib, R., Nilsson, L.-R., Kapur, S., Houle, E., Cabeza, R., and Mclntosh, A.R. (1995). Functional brain maps of retrieval mode and recovery of episodic information. Neuroreport 7, 249-252.

Ojemann, J.G., Akbudak, E., Snyder, A.Z., McKinstry, R.C., Raichle, M.E., and Conturo, T.E. (1997). Anatomic localization and quantitative analysis of gradient refocused echo-planar fMRI susceptibility artifacts. Neuroimage 6, 156-167.

Paivio, A., Yuille, J.C., and Madigan, S.A. (1968). Concreteness, imagery, and meaningfulness values for 925 nouns. J. Exp. Psychol. Monograph Suppl. 76 (No. 1).

Roediger, H.L. (1990). Implicit memory: Retention without remembering. Am. Psychol. 45, 1043-1056.

Roediger, H.L., and McDermott, K.B. (1993). Implicit memory in normal human subjects. In Handbook of Neuropsychology (Vol. 8), F. Boller, J. Grafman, eds. (New York: Elsevier Science).

Roediger, H.L., Weldon, M.S., and Challis, B.H. (1989). Explaining dissociations between implicit and explicit measures of retention: A processing account. In Varieties of Memory and Consciousness: Essays in Honour of Endel Tulving, H.L. Roediger and F.I.M. Craik, eds. (Lawrence Erlbaum Associates).

Rugg, M.D., Fletcher, P.C., Frith, C.D., Frackowiak, R.S.J., and Dolan, R.J. (1996). Differential activation of the prefrontal cortex in successful and unsuccessful memory retrieval. Brain 119, 2073-2083.

Sanders, A.L., Wheeler, M.E., and Buckner, R.L. (2000). Episodic recognition modulates frontal and parietal activity. J. Cogn. Neurosci. Suppl. 50A.

Schacter, D.L. (1994). Priming and multiple memory systems: Perceptual mechanisms of implicit memory. In Memory Systems 94, D.L. Schacter and E. Tulving, eds. (Cambridge: Bradford Book, MIT Press). Schacter, D.L., and Buckner, R.L. (1998). Priming and the brain. Neuron 20, 185-195.

Schacter, D.L., Alpert, N.M., Savage, C.R., Rauch, S.L., and Albert, M.S. (1996). Conscious recollection and the human hippocampal formation: Evidence from positron emission tomography. Proc. Natl. Acad. Sci. USA 93, 321-325.

Schacter, D.L., Buckner, R.L., Koutstaal, W., Dale, A.M., and Rosen, B.R. (1997). Late onset of anterior prefrontal activity during true and false recognition: An event-related fMRI study. Neuroimage 6 , 259-269.

Snyder, A.Z. (1996). Difference image vs. ratio image error function forms in PET-PET realignment. In Quantification of Brain Function using PET, D. Bailey and T. Jones, eds. (San Diego: Academic Press), pp. 131-137.

Stark, C.E.L., and Squire, L.R. (2000). Recognition memory and familiarity judgments in severe amnesia: no evidence for a contribution of repetition priming. Behav. Neurosci. 114, 459-467.

Talairach, J., and Tournoux, P. (1998). Co-planar stereotaxic atlas of the human brain. Stuttgart: Thieme.

Tulving, E., and Schacter, D.L. (1990). Priming and human memory systems. Science 24, 301-306.

Vaidya, C.J., Gabrieli, J.D., Keane, M.M., Monti, L.A., GutierrezRivas, H., and Zarella, M.M. (1997). Evidence for multiple mechanisms of conceptual priming on implicit memory tests. J. Exp. Psychol. Learn. Mem. Cogn. 23, 1324-1343.

Wagner, A.D., Gabrieli, J.D.E., and Verfaellie, M. (1997). Dissociations between familiarity processes in explicit recognition and im- 
plicit perceptual memory. J. Exp. Psychol. Learn. Mem. Cogn. 23, 305-323.

Wagner, A.D., Desmond, J.E., Glover, G.H., and Gabrieli, J.D.E. (1998). Prefrontal cortex and recognition memory: fMRI evidence for context-dependent retrieval processes. Brain 121, 1985-2002.

Wagner, A.D., Koutstaal, W., Maril, A., Schacter, D.L., and Buckner, R.L. (2000). Task-specific repetition priming in left inferior prefrontal cortex. Cereb. Cortex 10, 1176-1184.

Yonelinas, A.P., and Jacoby, L.L. (1996). Noncriterial recollection: Familiarity as automatic, irrelevant recollection. Conscious. Cogn. 5, 131-141.

Zarahn, E., Aguirre, G.K., and D'Esposito, M. (1997). Empirical analyses of BOLD fMRI statistics. I. Spatially unsmoothed data collected under null-hypothesis conditions. Neuroimage 5, 179-197. 